\title{
Experiments in Reconstructing Twentieth-Century Sea Levels
}

\author{
Richard D. Ray ${ }^{a}$, Bruce C. Douglas ${ }^{b}$ \\ ${ }^{a}$ NASA Goddard Space Flight Center, Greenbelt, Maryland, USA \\ ${ }^{b}$ Florida International University, Miami, Florida, USA
}

\begin{abstract}
One approach to reconstructing historical sea level from the relatively sparse tide-gauge network is to employ Empirical Orthogonal Functions (EOFs) as interpolatory spatial basis functions. The EOFs are determined from independent global data, generally sea-surface heights from either satellite altimetry or a numerical ocean model. The problem is revisited here for sea level since 1900. A new approach to handling the tide-gauge datum problem by direct solution offers possible advantages over the method of integrating sea-level differences, with the potential of eventually adjusting datums into the global terrestrial reference frame. The resulting time series of global mean sea levels appears fairly insensitive to the adopted set of EOFs. In contrast, charts of regional sea level anomalies and trends are very sensitive to the adopted set of EOFs, especially for the sparser network of gauges in the early 20th century. The reconstructions appear especially suspect before 1950 in the tropical Pacific. While this limits some applications of the sea-level reconstructions, the sensitivity does appear adequately captured by formal uncertainties. All our solutions show regional trends over the past five decades to be fairly uniform throughout the global ocean, in
\end{abstract}

Email address: richard.ray@nasa.gov (Richard D. Ray)

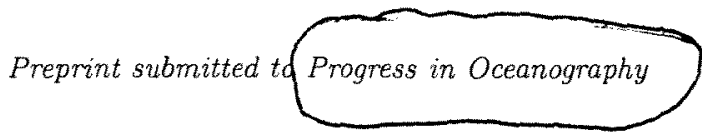

July 18,2011 
contrast to trends observed over the shorter altimeter era. Consistent with several previous estimates, the global sea-level rise since 1900 is $1.70 \pm 0.26$ $\mathrm{mm} \mathrm{yr}^{-1}$. The global trend since 1995 exceeds $3 \mathrm{~mm} \mathrm{yr}^{-1}$ which is consistent with altimeter measurements, but this large trend was possibly also reached between 1935 and 1950 .

Keywords: sea level, sea-level rise, sea-level reconstruction 